\title{
CHARACTERIZATIONS OF CONVEX SETS BY LOCAL SUPPORT PROPERTIES
}

\section{F. A. VAlentine}

It is our purpose to establish some new characterizations of convex sets by means of local properties and to derive as a consequence certain known results. This will be done for sets in a topological linear space $L$, such a space being a real linear space with a Hausdorff topology such that the operations of vector addition $x+y$ and scalar multiplication $\alpha x$ are continuous in both variables jointly [3]. The principal results are contained in Theorems 4 and 5. In order to describe matters simply, the following notations are used.

Notations. The interior, closure, boundary and convex hull of a set $S$ in $L$ are denoted by int $S$, cl $S$, bd $S$ and conv $S$ respectively. The closed line segment joining $x \in S$ and $y \in S$ is indicated by $x y$, whereas $L(x, y)$ stands for the line determined by $x$ and $y$. The interior of a set $S$ relative to the minimal linear variety containing it is denoted by intv $S$. Set union, intersection and difference are denoted by $\cup, \cdot$ and $\sim$ respectively. Vector addition and subtraction are denoted by + and - respectively. We let 0 and $\phi$ stand for the empty set and the origin of $L$ respectively.

In the statements of theorems and definitions the names of previous authors are indicated for historical purposes.

Definition 1. Let $S \subset L$. A point $x \in \mathrm{bd} S$ is called a point of mild convexity of $S$ if $x$ is not the midpoint of any segment uv with $0 \neq u v$ $\sim x \subset$ int $S$.

It is desirable to compare this definition with those given by Tietze [5] and by Leja and Wilkosz [4]. See also Kaufman [2]. For a brief summary of earlier results see Bonnesen and Fenchel [1, p. 7].

Definition 2. Let $x \in \mathrm{bd} S$, where $S \subset L$. The point $x$ is a point of weak or strong convexity of $S$, or a point of weak or strong concavity of $S$, if there exists a neighborhood $N(x)$ of $x$ and a linear functional $f$ with $f(x)=c$ such that the following conditions hold:

(a) (Tietze). The point $x$ is a point of weak convexity of $S$ if $f(y)>c$ with $y \in N(x) \sim x$ implies $y \notin S$. (For strong convexity replace $f(y)>c$ by $f(y) \geqq c$.

(b) (Leja and Wilkosz). The point $x$ is a point of strong concavity of $S$ if $f(y) \leqq c$ with $y \in N(x) \sim x$ implies $y \in S$. (For weak concavity replace $f(y) \leqq c$ by $f(y)<c$.)

Received by the editors April 20, 1959. 
As observed by Tietze [5], the following theorem of Leja and Wilkosz holds only in $L_{2}$, the two-dimensional normed linear space.

Theorem 1 (LeJa AND Wilkosz). Each open connected nonconvex set in $\mathrm{L}_{2}$ has at least one point of strong concavity (see Definition 2, (b)).

Hence, to remove the restriction to $L_{2}$, Tietze [5] proved the following theorem for sets in $L_{n}$, the finite $n$-dimensional case. It also holds in $L$.

Theorem 2 (TIETZE). Let $S$ be an open connected set in a topological linear space $L$. If each point of bd $S$ is a point of weak convexity of $S$ (see Definition 2, (a)), then $S$ is convex.

The concept in Definition 1 is a natural one for obtaining a form of the theorem of Leja and Wilkosz [4] for sets in $L$. The following Theorem 3 implies Theorem 2, whereas Theorem 1 implies Theorem 3 only for sets in $L_{2}$, the two-dimensional case.

Theorem 3. Let $S$ be an open connected set in a topological linear space $L$, and suppose each point $x \in \mathrm{bd} S$ is a point of mild convexity of $S$ (see Definition 1).

Then $S$ is convex.

Proof. Since $S$ is polygonally connected [3], to prove Theorem 3, it is sufficient to prove that $x z \subset S, z y \subset S$ implies $x y \subset S$. Let $H_{2}$ be a two-dimensional plane containing $x, y$ and $z$. Then let $K$ be the component of $S \cdot H_{2}$ containing $x, y$ and $z$. Theorem 1 , applied to $K$, implies that $K$ is convex, so that $x y \subset S$.

Note. A very short proof of Theorem 1 of Leja and Wilkosz exists, and it is given here for completeness. To do this, start as in the above paragraph, so that we merely need to show that $x y \subset S$. Suppose that $x y \llbracket S$. Let $S^{*}$ denote the set of those boundary points of $S$ which are in the triangle $\operatorname{conv}\left(x^{\prime} \cup y \cup z\right)$ where $x \in \operatorname{intv} x^{\prime} z$ and $x^{\prime} z \subset S$. Then conv $S^{*} \subset \operatorname{conv}\left(x^{\prime} \cup y \cup z\right)$. It is a very simple matter to show that there exists a point $p \in S^{*} \cdot \operatorname{conv} S^{*}$ which is an exposed point of conv $S^{*}$, i.e. there exists a line of support $L_{1}$ to conv $S^{*}$ such that $L_{1} \cdot \operatorname{conv} S^{*}=p$. Moreover, one can choose $p$ so that $p \notin x^{\prime} y$. Since $p \notin x^{\prime} z \cup z y \subset$ int $S$, and since $p$ is an exposed point of conv $S^{*}$, it is trivial to verify that $p$ is a point of strong concavity of $S$ (see Definition 2, (b)).

The following theorem extends Theorem 3 to connected sets without the assumption of openness.

Definition 3. A hyperplane $H$ strictly separates a set $S$ if each component of the complement of $H$ intersects $S$. The line $L_{1}$ pierces a set $S$ if 
each hyperplane containing $L_{1}$ strictly separates $S$.

THEOREM 4. Let $S$ be a closed connected set in a topological linear space $L$, with int conv $S \neq 0$. Assume that each point $x \in \mathrm{bd} S$ is a point of mild convexity of $S$ (see Definition 1). Also assume that each line $L_{1}$ through $x \in \operatorname{bd} S$ which pierces $S$ contains a segment $x y$ such that $0 \neq$ intv $x y \subset$ int $S$.

Then $S$ is convex.

Proof. Since int conv $S \neq 0$, let $u \in$ int conv $S$ and $v \in$ bd $S \sim u$. Clearly each hyperplane containing $u v$ must strictly separate $S$, otherwise $u \in$ bd conv $S$. Hence, by hypothesis, $v$ is linearly accessible from int $S$, so that int $S \neq 0$. Since $L$ is a topological linear space, each component of int $S$ is open. Let $K$ be a component of int $S$. Since each boundary point of $K$ is a boundary point of $S$, the set $K$ satisfies the hypotheses of Theorem 3 . Hence, $K$ is convex. Since $L$ is a topological linear space, the $\mathrm{cl} K$ is convex [3]. Suppose that $\operatorname{cl} K \not S$, and let $y \in S \sim \operatorname{cl} K$. Choose a point $z \in$ int $K$, and consider a two-dimensional plane $H_{2}$ containing $z y$. Let $H_{2} \cdot K \equiv C$, so that $C$ is a two-dimensional convex body, that is, intv $C \neq 0$. Since the set of points in bd $C$ at each of which there exists a unique line of support to $C$ is dense in bd $C$, there exists a point $x \in$ bd $C$ (sufficiently close to $y z \cdot \mathrm{bd} C$ ) through which a unique line of support $L_{1}$ to $C$ passes which strictly separates $y$ and $z$. Each hyperplane $H$ containing $L_{1}$ strictly separates $S$. This follows from the fact that $z \notin H$ if and only if $y \notin H$; also if $z \in H$, then $z \in \operatorname{int} S$ implies that $H$ separates $S$. Since bd $K \subset$ bd $S$, the hypothesis implies there exists a segment $x p \subset L_{1}$ such that $0 \neq$ intv $x p \subset$ int $S$. Since $(x+p) / 2 \in$ int $S$, relative to $H_{2}$, there exists a two-dimensional convex set $C_{1} \subset H_{2}$ such that $(x+p) / 2$ Cintv $C_{1}$, and such that $C_{1} \subset$ int $S$. Let $K_{1}$ be the component of int $S$ which contains $C_{1} \cup$ intv $x p$. Since, by Theorem 3 , each component of int $S$ is convex, the set $K_{1}$ is convex, and hence conv $\left(C_{1} \cup_{\text {intv }} x p\right)$ $C K_{1}$. However, since $L_{1}$ is the unique line of support to $C$ at $x$, and since intv $x p \subset K_{1} \cdot L_{1}$, it follows that $K \cdot K_{1} \neq 0$. This contradicts the fact that $K$ is a component of int $S$. Hence, $S=\operatorname{cl} K$, and the theorem is proved.

Definition 4. Let $S \subset L$ with $p \in S$. The set $S$ is said to have a radius of support relative to $p$ at each of its boundary points uniformly locally if the following holds: For each point $x \in$ bd $S$ there exists a neighborhood $N(x)$ such that for each point $y \in N(x) \cdot$ bd $S$ we have $S \cdot R(y, p)$ $\cdot[N(x)+y-x]=0$, where $R(y, p)$ is the relatively open half-line of the line $L(y, p)$ having endpoint $y$, and not containing $p$, and where $N(x)$ $+y-x$ is the translate of $N(x)$ to the point $y$. 
THEOREM 5. Let $S$ be a closed connected set in a topological linear space $L$, with int $S \neq 0$. Suppose that each point $x \in \mathrm{bd} S$ is a point of mild convexity of $S$ (see Definition 1). Also suppose there exists a point $p \in \operatorname{int} S$ such that $S$ has a radius of support relative to $p$ at each of its boundary points uniformly locally.

Then $S$ is convex.

Proof. In a topological linear space $L$, as a basis of fundamental neighborhoods of the origin $\phi$, it is always possible to restrict oneself to neighborhoods which are starshaped and centrally symmetric with respect to $\phi$. Since each translate and each nonzero scalar multiple of each neighborhood of $\phi$ is a neighborhood, we may restrict ourselves entirely to such neighborhoods [3]. We will do so throughout the following proof.

Let $K$ be that component of int $S$ which contains the point $p$. Since $K$ satisfies the hypotheses of Theorem 3 , the set $K$ is convex. Since $S$ is closed, the $\mathrm{cl} K$ is a convex subset of $S$, and bd $K \subset$ bd $S$. Suppose that cl $K \neq S$. Then since $S$ is connected, there exists a point $x \in \mathrm{bd} K$ which is a limit point of $S \sim \mathrm{cl} K$. Without loss of generality, assume that $x$ is the origin $\phi$. This may be accomplished without changing hypotheses by translating $S$ so that $x$ goes to the origin $\phi$. Let $V_{1}$ and $V_{2}$ be neighborhoods of $x=\phi$ (centrally symmetric and starshaped relative to $\phi)$ such that $V_{2}+V_{2} \subset V_{1}, V_{1}+V_{1} \subset N(\phi)$. Since $p \in$ int $K$, we have intv $p \phi \subset$ int $K$. Choose a point $q \in$ (intv $p \phi) \cdot V_{2}$. Let $U$ be a neighborhood of $q$ contained in $V_{2} \cdot K$ (centrally symmetric and starshaped relative to $q$ ). Let $V_{3} \equiv(U-q) \cdot V_{2}$, so that $V_{3}$ is a neighborhood of $\phi$. We have $V_{3} \subset V_{2}, V_{3}+q \subset V_{2}$. Since $\phi$ is a limit point of $S \sim \operatorname{cl} K$, there exists a point $y \in V_{3} \cdot(S \sim \operatorname{cl} K)$. Hence, $z \equiv y+q \in V_{3}+q$. Since the segment $q z \subset V_{3}+q$, we have $r \equiv p y \cdot q z \in V_{3}+q$. Since $V_{3}+q \subset V_{2} \cdot K$, we have $r \in V_{2} \cdot K$. Also $y \in V_{2} \sim \operatorname{cl} K$. Hence, let $r y \cdot($ bd $K) \equiv u$. Since $u=\lambda r+(1-\lambda) y$, where $0<\lambda<1$, and since $V_{2}+V_{2} \subset V_{1}$, we have $u \in V_{1}$. Since $-u \in V_{1}, y \in V_{1}$, we have $-u+y \in V_{1}+V_{1} \subset N(\phi)$. This implies that $y \in N(\phi)+u$. However, this contradicts the hypothesis that $u y \cdot(N(\phi)+u) \cdot(S \sim u)=0$, since $y \in S$. Hence, $S=\operatorname{cl} K$, and the theorem is proved.

The uniformity of the local radial support property in Theorem 5 cannot be omitted, for consider the following set $S \subset E_{2}$, where $E_{2}$ is the Euclidean plane with coordinates $\left(x_{1}, x_{2}\right)$. Let $S_{1}$ $=\left[\left(x_{1}, x_{2}\right): x_{1}^{2}+x_{2}^{2} \leqq 1\right]$ and $S_{2}=\left[\left(x_{1}, x_{2}\right): x_{1}^{2}+\left(x_{2}-2\right)^{2}=1\right]$. Now let $S=S_{1} \cup S_{2}, p=(0,0)$. The set $S$ satisfies all the hypotheses of Theorem 5 , except the radial support property is not uniform on account of the point $(0,1)$. 
Definition 5 (Tietze, see Definition 2, (a)). If $x$ is a point of weak convexity of a set $S \subset L$, all those points $y \in L$ for which $f(y)>C$, $y \in N(x)$ form a half-cell of support to $S$ at $x$.

Corollary to Theorem 5 ( $A$ generalization of a theorem of Tietze [5]). I. Suppose that $S$ is a closed connected set in a topological linear space $L$, and suppose int $S \neq 0$.

II. Suppose for each point $x \in$ bd $S$ there exists a neighborhood $N(x)$ of $x$ such that for each point $y \in N(x) \cdot b d S$, there exists a half-cell of support of $N(x)+y-x$ to $S$ at $y$.

Then $S$ is convex.

Proof. Hypothesis II of this corollary implies the last hypotheses of Theorem 5.

REMARK. If in hypothesis II of the corollary we assume that $N(x)=N(\phi)+x$, where $N(\phi)$ is a neighborhood of the origin $\phi$, then we obtain for $L$ the simplest generalization of the theorem of Tietze [5]. If $S$ is locally compact, we may weaken hypothesis II in the the corollary so that it holds on a dense subset of bd $S$, yielding as a result a theorem for sets in $L_{n}$ of the type studied by Kaufman [2].

\section{REFERENCES}

1. T. Bonnesen and W. Fenchel, Theorie der konvexen Körper, Ergebnisse der Mathematik und ihrer Grenzgebiete, vol. 3, no. 1, Berlin, Springer, 1934.

2. B. Kaufman, Über die Konvexitäts und Konkavitätstellen auf Jordankurven, J. Reine Angew. Math. vol. 164 (1931) pp. 112-127. 466.

3. V. L. Klee, Convex sets in linear spaces, Duke Math. J. vol. 18 (1951) pp. 443-

4. F. Leja and W. Wilkosz, Sur une propriête des domaines concaves, Ann. Soc. Polon. Math. vol. 2 (1924) pp. 222-224.

5. H. Tietze, Bemerkungen über konvexe und nicht-konvexe Figuren, J. Reine Angew. Math. vol. 160 (1929) pp. 67-69.

University of California at Los Angeles 